\title{
Author Index to Volume 29
}

Abrams SA, 615

Adamson GM, 496

Adamson TM, 381

Adlerberth I, 396

Aggett PJ, 297

Alaupovic P, 315

Albini $\mathrm{CH}, 619$

Alexander BJ, 110

Alpan G, 569

Amidon GL, 500

Anast CS, 201

Anderson GJ, 601

Andersson S, 56

Antila KJ, 272

Antonio-Santiago MT, 534

Aperia A, 263

Aquila WJ, 191

Aramburo MJ, 282

Argyle JC, 256

Armon $Y, 362,575$

Arnold BW, 110

Asayama K, 487

Ashkenazi S, 14

Avner ED, 151

Ayrault-Jarrier M, 315

Baan J, 473

Badoual J, 492

Balaraman V, 278

Bandini LG, 70

Bannon P, 123

Baraldi E, 575

Barbey-Morel C, 133

Barclay SM, 297

Barks JDE, 558

Barrington K, 372

Bartlett K, 406

Bartolome JV, 182

Baumgartner R, 403

Bauminger ER, 119

Beekman RH, 500

Bekaert ED, 315

Bendit I, 268

Bennett S, 329

Benson Jr, DW, 338

Bernstein D, 323

Berry SA, 510

Binoux M, 219

Bissonnette JM, 483

Björkhem I, 64

Black DD, 32

Blackett P, 315

Blanco CE, 586

Bland RD, 357

Boekkooi PF, 564

Böhler T, 128

Bortolussi R, 400

Brickell PM, 412

Brook CGD, 412

Brown EM, 20

Brusilow SW, 147

Burbridge S, 400

Burnell EE, 93, 98

Butzner JD, 594

Cairo MS, 504

Carlsson B, 396

Carlton DP, 357

Carter PA, 226

Cassidy SC, 466
Castro B, 417

Castro R, 342

Celniker A, 619

Cha C-JM, 606

Chang HK, 82

Chapman DL, 357

Chappell BA, 93, 98

Chen B, 191

Chen T, 110

Chen WW, 141

Chinoy MR, 534

Christensen RD, 1

Clark EB, 334

Clark RH, 160

Claybaugh JR, 278

Cleary TG, 14

Clyman RI, 569

Cochrane CG, 460

Cohen MB, 424

Connor WE, 601

Connuck DM, 334

Contrino J, 123

Cooper DM, 362, 575

Cope M, 553

Crane C, 323

Crowley MR, 167

Cummings JJ, 357

Cuneo B, 338

Curstedt T, 178

Daffos F, 219

Dalinghaus M, 466

D'Athis P, 492

Davidheiser S, 611

Davidson D, 538

Day L, 214

Dealler SF, 78

Deayton JM, 381

De Goeij JJM, 391

deLemos RA, 160

de Ley L, 178

Delpy DT, 553

Dietz WH, 70

DiPietro JA, 231

Dobashi K, 487

Donovan SM, 50

Drulis JM, 435

Ducros G, 548

Duffty P, 297

Durand M, 82

Durnford P, 400

Edwards AD, 553

Eisenfeld L, 123

El-Bizri H, 187

Eldemerdash A, 538

Ellis RW, 10

Ervin MG, 214, 342

Esteban NV, 615

Fineman JR, 167

Fine RN, 155

Finer N, 372

Fisher DA, 342

Fleming LR, 110

Fomon SJ, 435

Forestier F, 219

Frankenne F, 219

Frank L, 292, 376

Frazzini V, 611
Fresco R, 417

Fuhrman BP, 89

Fujieda K, 196

Fukagawa NK, 70

Fyfe JC, 24

Gall DG, 594

Ganzuka M, 353

Garcia RF, 256

Gärtner J, 14

Garza C, 243

Gauthier C, 529

Gelardi NL, 606

Gerstmann DR, 160

Gest AL, 623

Getman CE, 329

Giger U, 24

Gillis S, 504

Gilsdorf JR, 420

Goetzman BW, 329

Gore JC, 191

Greca N, 123

Greer NL, 182

Grönlund JU, 272

Grossmann G, 353

Gruppuso PA, 226

Guest I, 187

Gustafsson J, 64

Habib DM, 93, 98

Hachey DL, 243

Hall CA, 24

Hallman M, 56, 460

Hammerman C, 282

Hanson LA, 396

Hardouin S, 219

Harman AW, 496

Hashimoto T, 60

Hayashibe H, 487

Heldt G, 460

Henry JF, 243

Herson VC, 123

Hintz RL, 50

Hirao T, 455

Hoey K, 460

Hohimer AR, 483

Hopwood N, 619

Horn S, 119

Hosoda A, 196

Hosoi S, 455

Houghten RA, 460

Howland DF, 89

Hoy CM, 78

Hughes SF, 338

Hultgårdh-Nilsson A, 263

Humme JA, 93, 214

$\mathrm{Hu} \mathrm{N}, 334$

Hutchens TW, 243

Hyman PE, 20

Ichimura WM, 278

Inoue $Y, 455$

Inui S, 353

Jackson S, 406

Janghorbani M, 435

Jensen NJ, 424

Jezyk PF, 24

Johanson A, 619
Johnson CE, 500

Johnson JP, 525

Johnson NB, 510

Johnston V, 611

Kain ZN, 534

Kalli ST, 272

Kase BF, 64

Kato K, 487

Katz M, 119

Kawaoi A, 487

Keller BB, 334

Kelley RI, 141

Kepka DM, 151

Kikawa Y, 455

Kim HY, 440

Kim SY, 385

King T, 256

Kinsella JP, 160

Kitagawa M, 39

Klautz R, 473

Kliegman RM, 611

Klumpp SA, 24

Knight-Gibson C, 315

Kobayashi M, 487

Kobayashi T, 353

Koenig JM, I

Kohno Y, 25i

Kojima M, 5

Kok JSM, 381

Kölare S, 263

Kopelman H, 529

Kostyshak DA, 500

Koup R, 417

Krause PJ, 123

Kreutzer DL, 123

Kullama LK, 278

Lagercrantz H, 369

Laidlaw S, 155

Land J, 406

La Polla RJ, 460

LaRocco M, 14

Larsson SH, 263

Lassarre C, 219

Leake RD, 342

LeBoeuf RC, 155

Lechner AJ, 288

Lee P-C, 46

Leonard JV, 406

Levene MI, 78

Levine JS, 24

Levy J, 417

Lewiston NJ, 525

Liechty KW, 1

Linderkamp O, 128

Lin DS, 601

Lin E, 569

Lin $Y, 288$

Lloyd DJ, 297

Lodinová-Zádníková R, 396

Lopez EL, 14

Louie E, 525

Luthman J, 263

MacGillivray MH, 619

Mackay PM, 78

Maderazo EG, 123

Maeda K, 60

Marchitelli RN, 534 
Martin CG, 110

Martinez AM, 93, 98

Matsuo M, 173

Matsuura N, 196

Mauss D, 504

May L, 14

Mayumi M, 5

Mazor D, 119

McAuliffe JJ, 580

McCormick DC, 553

McDonnell WM, 420

McMicken HW, 623

McMillen IC, 381

Mehta P, 446

Mellander L, 396

Merritt TA, 460

Meyers RL, 347, 569

Meyerstein N. 119

Mezoff AG, 424

Mihalik SJ, 141

Miki M, 39

Millar MR, 78

Mills BJ, 619

Milner JA, 440

Mino M, 39

Mitchell MD, 1

Miyakawa Y, 5

Moak JP, 104

Moïse AA, 623

Moldwin R, 268

Moran C, 492

Morris Jr, SM, 151

Morrow WR, 160

Moser HW, 141

Motil KJ, 243

Motoike T, 173

Moxon ER, 406

Mullis PE, 412

Mura T, 251

Nakai A, 455

Nakamura H, 173

Nakamura KT, 278

Neijens HJ, 306

Nelson P, 155

Nelson SE, 435

$\mathrm{Ng} P C, 78$

Nguyen T, 500

Nisen PD, 268

Nitta K, 353

Norstein J, 543

Nowak R, 38

Null Jr, DM, 160

O'Brien WE, 151

Oetomo SB, 178

Ogihara T, 39

Ohashi T, 173

Oh D-M, 500

Oh W, 606
Okamoto H, 5

Okamoto R, 39

Okken A, 178

Okuno A, 196

Olive G, 492

Orii T, 60

Oshima M, 60

Pabst R, 237

Padbury JF, 93, 98

Panitch HB, 446

Papadimitriou JM, 496

Papouli M, 78

Pariente-Khayat A, 492

Passwell JH, 429

Patel MS, 412

Patterson DF, 24

Paulick RP, 347

Payne BD, 466

Pedersen JI, 64

Peeters LLH, 564

Perlmutter DH, 133

Persson H, 369

Pescovitz OH, 510

Petroff OAC, 191

Petschow BW, 208

Picciano MF, 440

Pichoff BE, 278

Pitkänen 0,56

Plunkett JM, 504

Pollitt RJ, 406

Pons $\mathrm{G}, 492$

Porges SW, 231

Posillico JT, 201

Post M, 558

Potter A, 553

Poulain FR, 357

Przbylski RJ, 61

Quattrin T, 619

Querfeld U, 155

Raff HV, 310

Raju TNK, 385

Revak SD, 460

Rey E, 492

Reynolds EOR, 553

Richard M-O, 492

Rich MA, 268

Rijkers GT, 306

Robbins J, 580

Robertson B, 353

Robinson G, 429

Rohwer-Nutter PL, 32

Romero R, 1

Rosenfeld RG, 50

Ross MG, 214, 342

Rothkötter HJ, 237

Rowley A, 417

Rubens CE, 310
Rubin LJ, 449

Rubin LP, 201

Rudolph AM, 347, 466

Rudolph CD, 347

Ruef P, 128

Saito M, 455

Salusky IB, 155

Sanderud J, 543

Sano K, 173

Saugstad OD, 543

Saxena PR, 564

Scarpelli EM, 534

Schanberg SM, 182

Schanler RJ, 243

Scharenberg JJM, 306

Schellekens H, 400

Schoeller DA, 70

Schoots C, 178

Schreyer P, 214

Sejersen T, 263

Shaffer TH, 446

Shahal Y, 119

Sherman B, 619

Sherman DJ, 214

Shigematsu Y, 455

Shinn C, 302

Shinohara M, 302

Shiraki K, 251

Shuford W, 310

Shulman S, 417

Sieck GC, 586

Siimes ASI, 272

Slavíková M, 396

Snape Jr, WJ, 20

Soifer SJ, 167

Sosenko IRS, 292

Sotos J, 619

Spaapen LJM, 403

Srinivasan M, 369

Steendijk P, 473

Steinberg BM, 268

Struve MF, 46

Sudo M, 455

Sullivan $\mathbf{J}, 417$

Sullivan KJ, 82

Suzuki Y, 60

Svanborg C, 396

Sweeney Jr, WE, 15

Sydänmaa M, 272

Takada S, 173

Talbott RD, 208

Tamai H, 39

Taylor AF, 160

Teitel DF, 466, 473

Terazawa S, 5

ter Haar JG, 178

Thio SL, 93, 98

Tlaskalová-Hogenová H, 396
Tod ML, 449

Trippenbach T, 548

Tsuda F, 5

Tsuneishi S, 173

Tuor UI, 517, 558

Turnbull DM, 406

Uchida N, 487

Ulbrich $\mathrm{H}, 237$

Välimäki IAT, 272

van Bel F, 473

Van den Hamer CJA, 391

van der Meer SB, 403

van der Velde ET, 473

van de Ven C, 504

van Dongen JM, 306

Van Wouwe JP, 391

Varma DR, 187

Veldhuizen M, 391

Vella PP, 10

Venkataraman ST, 89

Verkeste CM, 564

Viera NE, 615

Volovitz B, 429

Waber P, 268

Walker TD, 226

Wallig MA, 440

Wathne K-O, 64

Weickmann PH, 623

Wendel U, 403

Werlin SL, 46

Wildevuur ChRH, 178

Wilson DM, 50

Wilson GN, 256

Wine JJ, 525

Wold A, 396

Wolff E, 310

Wolfson MR, 446

Woodhead JC, 435

Wyatt JS, 553

Wykes LJ, 70

Yagi H, 20

Yamaguchi S, 60

Yamamoto Y, 369

Yamanaka T, 5

Yasuda H, 39

Yeager AM, 302

Yergey AL, 615

Yip T-T, 243

Yoshimura K, 449

Yotsumoto $\mathrm{S}, 5$

Young RSK, 191

Yuri K, 196

Zanconato S, 362, 575

Zegers BJM, 306

Zimmerman FJ, 338

Zmora E, 119 


\section{Subject Index to Volume 29}

Aciduria, glutaric, electron transfer flavoprotein deficiency in patients with, 60

Adolescents, handicapped, body composition and energy expenditure, 70

Adrenergic agonist, hemodynamic response, during chronic hypoxemia, neonatal lamb, 323

Age

enterohemorrhagic Escherichia coli adherence and, rabbit intestinal cells, 14

paracetamol toxicity and, postnatal mice 496

transdermal electrical potential and, 78

Air trapping, pulmonary vascular resistance and, neonatal lamb, 89

Airway, smooth muscle, xanthine derivative effect, preterm lamb, 446

Aminophylline, intrapulmonary, lung liquid decreased by, fetal lamb, 357

Amniotic fluid, interleukin-6 in, possible sources, 1

$\alpha_{1}$-Antitrypsin, effect of Pseudomonas elastase, mononuclear phagocytes, 133

Apnea, appearance of, preterm infants, 372

ApoA-I, lipoprotein particles containing, composition, sex- and age-related differences, 315

Apolipoprotein, intestinal synthesis, newborn piglet, 32

Arginine, biosynthetic enzymes, localization, renal proximal tubules, mice, 151

Asthma, atopic, urinary excretion of leukotriene $\mathrm{E}_{4}, 455$

Atherosclerosis, lipoprotein plasma levels and, continuous peritoneal dialysis, 155

Atrial natriuretic factor, ring-deleted analogue, renal and cardiovascular effects, ovine fetus, 342

Bifidobacterium, response to growth promoters, human and cow milk, 208

Birth, activation of preprotachykinin-A mRNA, respiratory neural structures, rabbit, 369

$\beta$-Blocker, cardiorespiratory control and, neonatal lamb, 272

Blood flow

autoregulation, during hypotension and hypertension, infant lamb, brain, 110

cerebral, developmental changes, newborn rabbit, 517

left ventricular output, effect of in utero ventilation, fetal sheep, 466

regional, ductus venosus obstruction effect, fetal lamb, 347

uteroplacental, nondeformable red blood cell effect, pregnant guinea pig, 564

Blood volume, cerebral, arterial carbon dioxide tension changes and, neonate, 553

Body composition, adolescents with cerebral palsy or myelodysplasia, 70

Body water, effect of plasma protein reduction, fetal sheep, 623

Brain

blood flow

autoregulation, during hypotension and hypertension, infant lamb, 110

developmental changes, newborn rabbit, 517 energy state, lactate metabolism and, status epilepticus, neonatal dog, 191

Brain damage, hypoxic-ischemic, dexamethasone prevention, neonatal rat, 558

Breastfeeding, antibody response to artificial E. coli colonization, comparison, nonbreast-fed infants, 396

Breast milk: see Milk, human

Bronchopulmonary dysplasia

intubated infants, pulmonary function test for, 82

pulmonary hypertension with, nifedipine pharmacokinetics and pharmacodynamics, 500

Busulfan, high-dose, hematopoietic cell transplantation after, twitcher mouse, 302

Caffeine

acetylation, maturation of, infants, 492

effect on airway smooth muscle, preterm lamb, 446

Calcium, absorption and endogenous fecal excretion, low birth weight infants, 615

Calcium antagonist, vascular responses, differential ontogeny, rat, 278

Carbon dioxide

arterial tension, cerebral blood volume response to changes in, neonate, 553

cerebral vascular reactivity, effect of hematocrit alterations, neonatal baboon, 385

Cardiac cycle length, effect on ventricular end-diastolic pressure, stage 24 chick embryo, 338

Cardiac output, diastolic filling characteristics, stage 12 to 27 chick embryo ventricle, 334

Catecholamines, myocardial receptor density and, newborn sheep, 98

Cell transplantation, hematopoietic, after high-dose busulfan, twitcher mouse, 302

Cerebral palsy, body composition and energy expenditure, adolescents, 70

Chenodeoxycholic acid, formation, peroxisome role, human liver, 64

Chloride, efflux, cAMP-sensitive, rabbit pancreatic acini, 529

Circulation: see Bloodflow

Clofibrate, maternal administration, effect on fetal peroxisomes, mice, 256

Cobalamin, inherited selective intestinal malabsorption, clinical, metabolic, pathologic and genetic features, dog, 24

Cocaine

fetal exposure

enhanced lung maturation after, rabbit, 534

teratogenic effects, rat, 187

Colon, smooth muscle contraction, agonistmediated, developmental changes, rabbit, 20

Cystic fibrosis

chloride efflux pathway, rabbit pancreatic acini, 529

heterozygote, $\beta$-adrenergic sweat responses, 525
Deoxyribonucleic acid, synthesis, human milk effect, neonatal rat hepatocytes, 251

Dexamethasone

effect on pulmonary surfactant metabolism, hyperoxia-treated rat lung, 173

prevention, hypoxic-ischemic brain damage, neonatal rat, 558

Diabetes, streptozotocin-induced, neonatal macrosomia, cross-generation effect, rat pup, 606

Dialysis, continuous peritoneal, lipoprotein concentrations in, 155

Diaphragm, muscle fibers, succinate dehydrogenase activity, postnatal changes, cat, 586

Diarrhea, Escherichia coli heat-stable enterotoxin-mediated, increased susceptibility mechanisms, pig, 424

Diltiazem, vascular response, differential ontogeny, rat, 278

Dopamine

circulatory effects, cyanotic heart disease, neonatal lamb, 323

pulmonary and systemic circulatory effects, hypoxia-induced pulmonary hypertension, lamb, 329

Ductus venosus, obstruction, effect on liver and regional blood flow, fetal lamb, 347

Egg yolk, fatty acid composition, effect on essential fatty acid utilization, chick embryo, 601

Electron transfer flavoprotein, genetic deficiency, in glutaric aciduria type II, 60

Embryo

development, cocaine effect, rat, 187

essential fatty acid utilization, effect of egg yolk fatty acid composition, chick, 601

heart ventricle, diastolic filling characteristics, chick, 334

$\beta$-Endorphin, central administration, effect on lung ornithine decarboxylase activity, developing rat, 182

Endothelium-derived relaxing factor, pulmonary vascular resistance regulated by, neonatal guinea pig lung, 538

Energy expenditure, adolescents with cerebral palsy or myelodysplasia, 70

Enteritis, acute bacterial, refeeding effect, malnourished infant rabbit, 594

Erythrocyte: see Red blood cell

Escherichia coli

artificial colonization, antibody response, breast-fed versus non-breast-fed infants, 396

enterohemorrhagic, adherence to intestinal cells, age effect, rabbit, 14

heat-stable enterotoxin, increased susceptibility mechanisms, pig, 424

European Society for Paediatric Infectious Diseases, selected abstracts from 8 th annual meeting, 116

Exercise

burst, maturation of ventilatory responses to, 362 


\section{SUBJECT INDEX TO VOLUME 29}

high-intensity, heart rate recovery, children and adults, 575

urinary excretion of leukotriene $E_{4}$ after asthmatic children, 455

Fatty acid

essential, effect of egg yolk fatty acid composition, developing chick embryo, 601

long-chain, deficiency, clinical features and biochemical findings, 406

odd-numbered long-chain, inherited dis-

Fetus orders of proprionate metabolism, 403

body water content, effect of plasma protein reduction, sheep, 623

ductus venosus obstruction, effect on liver and regional blood flow, lamb, 347

incidence of breathing movements, vascular lactic acid infusion effect, sheep, 483

inherited disorder of proprionate metabolism, odd-numbered long-chain fatty acid accumulation, 403

interleukin- 6 production, during intraamniotic infection, 1

left ventricular output, effect of in utero ventilation, sheep, 466

lung liquid absorption, intrapulmonary terbutaline and aminophylline effect, lamb, 357

lung maturation, cocaine effect, rabbit, 534

normal and pressure-loaded heart, troponin $\mathrm{T}$ expression, sheep, 580

peroxisome proliferation, maternal clofibrate treatment effect, mice, 256

pulmonary vascular resistance, decreases, indomethacin prevention, lamb, 449

rehydration, via amniotic infusion, fetal swallowing role, sheep, 214

renal and cardiovascular function, atrial natriuretic factor analogue effect sheep, 342

serum insulin-like growth factors, growth/ growth retardation related to, 219

Galactosylceramidase, deficiency, high-dose busulfan treatment effect, mice, 302

Gavage feeding, vagal responsiveness to, as index of preterm status, 231

Gender, iron absorption and, preadolescent children, 435

Gene expression, preprotachykinin-A, perinatal ontogeny, respiratory neural structures, rabbit, 369

Gene mutation, variance in sweat responses and, cystic fibrosis heterozygotes, 525

Globoid cell, leukodystrophy, high dose busulfan effect, mice, 302

Glucocorticoid

protection, cerebral hypoxia-ischemia, neonatal rat, 558

regulation of lingual lipase development by, suckling rat, 46

Gonadotropin, pulsatile secretion, age-related change, Turner syndrome, 196

Granulocyte-monocyte colony stimulating factor, modulation of neonatal myelopoiesis by, neonatal rat, 504

Growth and development

age and enterohemorrhagic Escherichia coli adherence, rabbit intestinal cells, 14

apoA-I-containing lipoprotein particles, children and adults, 315

burst exercise, maturation of ventilatory responses to, 362 circadian sleep-wake rhythms, preterm and full-term infants, 381

cocaine effect, rat embryo, 187

colonic smooth muscle contraction, agerelated changes, rabbit, 20

developmental changes, ventricular enddiastolic pressure, stage 24 chick embryo, 338

gene expression in renal proximal tubules, mice, 151

heart rate recovery from high-intensity exercise, children and adults, 575

hepatic type I insulin-like growth factor receptor, pre- and postnatal regulation, rat, 226

idiopathic growth failure, urinary growth hormone measurement in children with, 619

increased susceptibility to Escherichia coli heat-stable enterotoxin, immature and weaned pigs, 424

insulin-like growth factors and, normal and growth-retarded human fetus, 219

lingual lipase control during, glucocorticoid role, rat, 46

local cerebral blood flow, developmental changes, newborn rabbit, 517

lung maturation, central administration of $\beta$-endorphin effect, rat, 182

maturation of caffeine $\mathrm{N}$-acetylation, infants, 492

paracetamol toxicity, age-related differences in susceptibility, mice, 496

pulsatile gonadotropin secretion, age-related change, Turner syndrome, 196

regulation principles, postnatal changes, proximal tubule cells, infant and adolescent rat, 263

sodium nitroprusside and dopamine, agedependent effects, pulmonary hypertension, lamb, 329

superoxide dismutase histochemistry, fetal and neonatal rat lung and kidney, 487

transdermal potential of human skin, evolution of, 78

Growth failure, idiopathic, urinary growth hormone measurement in children with, 619

Growth hormone, urinary measurement, diagnostic significance, short children, 619

Growth hormone releasing hormone, messenger ribonucleic acid, ontogeny, rat placenta, 510

Gut, lamina propria, lymphocyte numbers and subsets, germ-free pigs, 237

Haemophilus influenzae type b

capsular polysaccharide, mucosal antibodies, 420

conjugate vaccine, immunogenicity, infant monkeys, 10

Heart

atrial natriuretic factor analogue effect, ovine fetus, 342

cyanotic disease, circulatory effects, isoproterenol versus dopamine, neonatal lamb, 323

normal and pressure-loaded, troponin $\mathrm{T}$ expression, fetal sheep, 580

Purkinje fibers, electrophysiologic effects of $d$-sotalol, dog, 104

vagal responsiveness to gavage feeding, as index of preterm status, 231

Heart rate, recovery, after high-intensity exercise, children and adults, 575

Heart ventricle contractility evaluation, end-systolic pressure-volume relationship, newborn lamb, 473

stage 24 , cardiac cycle length effect, chick embryo, 338

stage 12 to 27 , diastolic filling characteristics, chick embryo, 334

Hematocrit, alterations, cerebral vascular $\mathrm{CO}_{2}$ reactivity and, newborn baboon, 385

Hepatitis B, perinatal infection, role of $\mathrm{HBV}$ mutants in carrier mothers, 5

Hepatocyte, deoxyribonucleic acid synthesis, human milk effect, neonatal rat, 251

Heterozygote, advantage, sweat response variance and, cystic fibrosis, 525

Human growth hormone, gene cluster analysis, constitutionally short stature, 412

Hyaline membrane disease, hemodynamic effects of high frequency oscillatory ventilation, comparison, intermittent mandatory ventilation, premature baboon, 160

3-Hydroxyacyl-coenzyme A dehydrogenase, long-chain, deficiency, clinical features and biochemical findings, 406

Hyperoxia

antioxidant enzymes during, premature and term rabbits, 292

neonatal tolerance, premature weaning and, rat pup, 376

pulmonary surfactant accumulation and, dexamethasone effect, rat lung, 173

selenium role, neonatal rat lung, 440

Hypertension

autoregulation of brain blood flow during, infant lamb, 110

pulmonary

age-dependent effects, sodium nitroprusside and dopamine, lamb, 329

GBS-induced, hyperventilation after, piglet, 282

vasoactive drug effect, neonatal lamb, 167

Hyperventilation, prostacyclin formation and, after GBS-induced pulmonary hypertension, piglet, 282

Hypotension, autoregulation of brain blood flow during, infant lamb, 110

Hypoxemia

hemodynamic response to adrenergic agonists during, comparison, isoproterenol and dopamine, neonatal lamb, 323

inhibition of fetal breathing movements during, vascular lactic acid infusion effect, sheep, 483

Hypoxia

cerebral, dexamethasone protection, neonatal rat, 558

pulmonary hypertension induced by, agedependent effects, sodium nitroprusside and dopamine, lamb, 329

Immunodeficiency disease, severe combined, abnormal signal transduction in patient with, 306

Immunoglobulin A

polyribosylribitol phosphate-specific antibodies, saliva, patients with Haemophilus influenzae type b infection, 420

secretory, receptor identification, breastmilk macrophages, 429

Immunoglobulin $\mathrm{M}$, monoclonal antibody, pharmacokinetics, neonatal monkey, 310

Indomethacin, patent ductus arteriosus 
treated with, terminal ileum ischemia Infant and, fetal lamb, 569

acute bacterial enteritis, refeeding effect, malnourished rabbit, 594

brain blood flow, autoregulation during hypotension and hypertension, lamb, 110

low birth weight

calcium absorption and endogenous fecal excretion, 615

iron supplementation, 297

premature

free radicals in lipid emulsion, 56

human milk-fed, intact lactoferrin in urine of, 243

Infection, intraamniotic, interleukin-6 production during, fetal and maternal cells, 1

Insulin, receptor, number and binding affinity, neonatal dog, 611

Insulin-like growth factor

binding proteins, growth/growth retardation related to, fetus, 219

measurement, rat milk, 50

messenger ribonucleic acid, ontogeny, rat placenta, 510

serum concentrations, growth/growth retardation related to, fetus, 219

type I receptor, ontogeny, rat liver, 226

Insulin-like growth factor-I, gene analysis, constitutionally short stature, 412

Interferon, effect, neonatal Listeria monocytogenes infection, rat, 400

Interleukin-3, modulation of myelopoiesis by, neonatal rat, 504

Interleukin-6, production, fetal and maternal cells, during intraamniotic infection, 1

Intestine

artificial E. coli colonization, antibody response, breast-fed versus non-breastfed infants, 396

enterohemorrhagic Escherichia coli adherence to, postnatal age effect, rabbit, 14

Intraamniotic fluid, fetal rehydration and, Iron fetal swallowing role, sheep, 214

absorption, gender effect, preadolescent children, 435

supplementation, reduced erythrocyte superoxide dismutase activity and, 297

Ischemia

cerebral, dexamethasone protection, neonatal rat, 558

intestinal, patent ductus arteriosus, indomethacin, and intestinal distension as risk factors for, fetal lamb, 569

Isoproterenol

circulatory effects, cyanotic heart disease, neonatal lamb, 323

left ventricular response, effect of in utero ventilation, fetal sheep, 466

Jugular vein, effect of lactic acid injection, neonatal rabbit, 548

Kawasaki syndrome, lack of retroviral etiology for, 417

Kidney

atrial natriuretic factor analogue effect, ovine fetus, 342

development and oncogenesis, N-myc oncogene expression, pig, 268

proximal tubule

arginine biosynthetic enzyme localization, mice, 151 postnatal growth and C-fos expression, infant and adolescent rat, 263

superoxide dismutase perinatal development, immunohistochemical study, rat, 487

Lactate, metabolism, brain energy state and during status epilepticus, neonatal dog, 191

Lactic acid

injection, jugular vein, neonatal rabbit.

vascular infusion, effect on incidence of fetal breathing movements, sheep, 483

Lactoferrin urinary excretion, human milk fed preterm infants, 243

Lamina propria, postnatal lymphocyte de velopment, conventional and germfree pigs, 237

Leukocyte

deformability and volume, neonate and adult, 128

polymorphonuclear, pentoxifylline effect, neonate, 123

Leukotriene $\mathrm{E}_{4}$, urinary excretion, exerciseinduced, asthmatic children, 455

Lingual lipase, development, modulation by glucocorticoid, rat, 46

Lipid, dietary and biliary absorption, intestinal apolipoprotein synthesis and, newborn piglet, 32

Lipid emulsion, parenteral, generation of free radicals in, 56

Lipoprotein

apoA-I-containing, composition, children and adults, 315

neonatal, susceptibility to oxidative stress, 39

plasma concentrations, continuous peritoneal dialysis, 155

Listeria monocytogenes, neonatal infection. interferon treatment, rat, 400

Liver

lood flow, ductus venosus obstruction effect, fetal lamb, 347

neonatal growth, human milk effect, rat hepatocyte culture, 251

paracetamol toxicity, susceptibility, developmental aspects, mice, 496

type I insulin-like growth factor receptor, ontogeny, rat, 226

Lung

developing tissue, selenium role, neonatal rat, 440

edema fluid, inactivation of exogenous surfactant by, neonatal rabbit, 353

hypertension, hyperventilation effect, piglet, 282

liquid, effect of intrapulmonary terbutaline and aminophylline, fetal lamb, 357

maturation, cocaine-exposed fetus, rabbit, 534

mechanical function, assessment, intubated infants, 82

ornithine decarboxylase activity, effect of central $\beta$-endorphin administration, developing rat, 182

oxygen toxicity, premature versus term rabbits, 292

pulmonary vasoconstriction, reactive oxygen metabolite role, young pig, 543

regulation of pulmonary vascular resistance, neonatal guinea pig, 538

role, norepinephrine clearance, lamb, 93

superoxide dismutase perinatal development, immunohistochemical study, rat, 487 surfactant content and type II cell devel opment, during prenatal starvation fetal guinea pig, 288

synthetic pulmonary surfactant formation, synthetic peptide use, 460

Lymphocyte, postnatal development, gut lamina propria, conventional and germ-free pigs, 237

Macrophage, breast-milk, secretory immunoglobulin A receptor on, 429

Macrosomia, neonatal, cross-generation ef fect, rat pups of diabetic mothers, 606

Malnutrition, protein-energy, refeeding effect during acute enteritis, infant rabbit 594

Mechanical ventilation, pulmonary vascular resistance decreased by, indomethacin effect, fetal lamb, 449

Messenger ribonucleic acid

abundance, during kidney development, mice, 151

growth hormone releasing hormone and IGF-I and II. ontogeny, rat placenta, 510

preprotachykinin-A, birth related activation, respiratory neural structures, rabbit, 369

Microcirculation, leukocyte deformability and volume, neonate, 128

Milk

cow, growth promoters, Bifidobacterium response to, 208

human

macrophage, secretory immunoglobulin A receptor on, 429

physiologic role, liver growth, rat hepatocyte culture, 251

growth promoters, Bifidobacterium response to, 208

rat, role of insulin-like growth factors, 50

Monoclonal antibody, human IgM, pharmacokinetic and pharmacodynamic analysis, neonatal monkey, 310

Muscle contraction, colonic, ontogeny of rabbit, 20

Muscle fiber, succinate dehydrogenase activity, postnatal changes, cat, 586

Myelodysplasia, body composition and energy expenditure, adolescents, 70

Neonate

adaptation, $\beta$-adrenergic receptors at birth, sheep, 98

arterial carbon dioxide tension, changes, cerebral blood volume response, 553

cardiorespiratory control, effect of $\beta$-adrenergic blockade and sleep state, Jamb, 272

cerebral vascular $\mathrm{CO}_{2}$ reactivity, hematocrit alterations effect, baboon, 385

circadian sleep-wake rhythms, development, preterm and full-term infants, 381

cyanotic heart disease, comparative circulatory effects, isoproterenol versus dopamine, lamb, 323

end-systolic pressure-volume relationship, effects of loading and inotropic interventions, lamb, 473

erythrocytes, oxidative stress, 119

fulminant hepatitis $\mathrm{B}$, role of $\mathrm{HBV} \mathrm{mu}-$ tants in carrier mothers, 5

human IgM monoclonal antibody treatment, bacterial infection, monkey, 310

hypoxic-ischemic brain damage, dexa- 
methasone prevention, rat, 558

inherited disorder of proprionate metabolism, odd-numbered long-chain fatty acid accumulation, 403

insulin resistance, insulin receptor binding defect and, dog, 611

intestinal apolipoprotein synthesis, effect of dietary and biliary lipid absorption, piglet, 32

lactic acid injection, jugular vein, rabbit, 548

leukocyte, deformability and volume, 128

lipoproteins, peroxidizability of, 39

Listeria monocytogenes infection, interferon treatment, rat, 400

local cerebral blood flow, developmental changes, autoradiographic study, rabbit, 517

lung development, selenium role, rat, 440

macrosomic, enhanced growth, cross-generation effect, neonatal rat, 606

mechanical ventilation, air trapping effect, lamb, 89

myelopoiesis, modulation, rat, 504

neutrophil, modulation by pentoxifylline, 123

parathyroid hormone, circulating levels, 201

pulmonary hypertension, vasoactive drug effect, lamb, 167

pulmonary vascular resistance, regulation by endothelium-derived relaxing factor, guinea pig, 538

respiratory distress syndrome, inactivation of exogenous surfactant by pulmonary edema fluid, rabbit, 353

status epilepticus, brain energy state and lactate metabolism, dog, 191

tolerance to hyperoxia, premature weaning and, rat pup, 376

Neutrophil, pentoxifylline effect, neonate, 123

Nifedipine

pharmacokinetics and pharmacodynamics, bronchopulmonary dysplasia and pulmonary hypertension, 500

vascular response, differential ontogeny, rat, 278

Nitroprusside, pulmonary and systemic circulatory effects, hypoxia-induced pulmonary hypertension, lamb, 329

Norepinephrine, pulmonary clearance, lamb, 93

Nutrition, early dietary, subclinical zinc deficiency, laboratory assessment, weaning rat, 391

Oncogene, expression, kidney development and oncogenesis, pig, 268

Ornithine decarboxylase, lung activity, effect of central administrationof $\beta$-endorphin, developing rat, 182

Oxygen

reactive metabolite, pulmonary vasoconstriction produced by, young pig, 543

toxicity, premature versus term rabbit lung, 292

Pancreas, acini, cAMP-sensitive chloride efflux, rabbit, 529

Paracetamol, toxicity, susceptibility, developmental aspects, mice, 496

Parathyroid hormone, circulating levels, neonate, 201

Parenteral nutrition, lipid emulsion, free radicals in, 56
Patent ductus arteriosus, indomethacin treatment, terminal ileum ischemia and, fetal lamb, 569

Pentoxifylline, modulation of neutrophil function by, neonate, 123

Peptide, synthetic, role, pulmonary surfactant formation, 460

Peroxisome

proliferation, maternal clofibrate administration effect, mice, 256

role, chenodeoxycholic acid formation, human liver, 64

Phagocyte, mononuclear, $\alpha_{1}$-antitrypsin expression, Pseudomonas elastase effect, 133

Phenylacetylglutamine, as vehicle for waste nitrogen excretion, 147

Phenylhydrazine, exposure, newborn erythrocytes, 119

Phosphodiesterase, inhibition, effect on fetal lung liquid secretion, lamb, 357

Placenta, ontogeny of growth hormone releasing hormone mRNA, rat, 510

Preadolescence, iron absorption, gender-related differences, 435

Pregnancy, uteroplacental blood flow, nondeformable red blood cell effect, guinea pig, 564

Prematurity: see also Infant, premature

airway smooth muscle, caffeine and theophylline effect, preterm lamb, 446

hyaline membrane disease, hemodynamic effects, high-frequency oscillatory ventilation, baboon, 160

hyperoxic exposure, antioxidant enzyme levels during, rabbit, 292

idiopathic apnea of, appearance, 372

index, vagal responsiveness to gavage feeding as, 231

surfactant instillation, distribution, lunglavaged rabbit, 178

Propionate metabolism, inherited disorder, prenatal odd-numbered fatty acids in, 403

Propranolol, cardiorespiratory control and, neonatal lamb, 272

Prostacyclin, mediation, hyperventilation-induced pulmonary vasodilation, piglet, 282

Protein

growth promotional activity, Bifidobacterium species, human and cow milk, 208

peroxisomal, pathogenesis of Zellweger syndrome, 141

plasma reduction, body water content and, fetal sheep, 623

Protooncogene, proliferative rate, postnatal growth effect, infant and adolescent rat, 263

Pseudomonas aeruginosas, elastase, effect on $\alpha_{1}$-antitrypsin expression, mononuclear phagocyte, 133

Pulmonary artery, pressure, hyperventilation effect, piglet, 282

Pulmonary surfactant

endotracheally instilled protein, distribution, lung-lavaged rabbit, 178

exogenous, inactivation by pulmonary edema fluid, neonatal rabbit, 353

fetal levels, during prenatal starvation, guinea pig, 288

metabolism, dexamethasone effect, hyperoxia-treated rat lung, 173

synthetic, synthetic peptide role, 460

Pulmonary vascular resistance

air trapping effect, neonatal lamb, 89

regulation by endothelium-derived relax- ing factor, neonatal guinea pig lung, 538

ventilation-induced decreases, indomethacin effect, fetal lamb, 449

Pulmonary vasoconstriction: see Vasoconstriction

Purkinje fibers, $d$-sotalol effect, canine heart, 104

Red blood cell

nondeformable, effect on uteroplacental blood flow, guinea pig, 564

oxidative stress, neonates, 119

superoxide dismutase activity, iron-supplemented low birth weight infants, 297

Refeeding, during acute bacterial enteritis, effect on small intestinal repair, malnourished infant rabbit, 594

Respiration, ventilatory response to burst exercise, maturation of, 362

Respiratory distress syndrome

exogenous surfactant therapy, inactivation by pulmonary edema fluid, neonatal rabbit, 353

intubated infants, pulmonary function test for, 82

Retrovirus, lack of, cultured lymphocytes, Kawasaki syndrome, 417

Selenium, neonatal lung development, rat, 440

Short stature, constitutionally, gene analysis, 412

Signal transduction, abnormal, severe combined immunodeficiency disease, 306

Skin, transdermal potential, development of,

Sleep state, cardiorespiratory control and, neonatal lamb, 272

Sleep-wake cycle, circadian, preterm and fullterm infants, 381

$d$-Sotalol, developmental cellular electrophysiologic effects, cardiac Purkinje fibers, dog, 104

Starvation, prenatal, surfactant content during, fetal lung, guinea pig, 288

Status epilepticus, brain energy state and lactate metabolism during, neonatal dog, 191

Stress

oxidative, susceptibility, neonatal lipoproteins, 39

preterm, cardiac vagal tone during gavage feeding, 231

Succinate dehydrogenase, activity distribution, postnatal changes, diaphragm muscle fibers, cat, 586

Superoxide dismutase

erythrocyte activity, iron-supplemented low birth weight infants, 297

perinatal development, immunohistochemical study, rat lung and kidney, 487

Surfactant: see Pulmonary surfactant

Terbutaline, intrapulmonary, lung liquid decreased by, fetal lamb, 357

Theophylline, effect on airway smooth muscle, preterm lamb, 446

Transient tachypnea syndrome, acidemia role, neonatal rabbit, 548

Troponin T, expression, normal and pressure-loaded fetal sheep heart, 580

Turner syndrome, pulsatile gonadotropin secretion, age-related change, 196 
Urea, inborn errors of synthesis, waste nitrogen excretion, phenylacetylglutamine role. 147

Urine, excretion of matcrnal lactoferrin, human milk-fed preterm infants, 243

Vaccine, Haemophilus influenzae type b conjugate, immunogenicity, infant monkeys, 10

Vasoactive drugs, hemodynamic effects, pulmonary hypertension, neonatal lamb. 167
Vasoconstriction, pulmonary, reactive oxygen metabolite role, young pig, 543

\section{Ventilation}

high-frequency oscillatory, hemodynamic cffects, premature baboon, 160

mechanical: see Mechanical ventilation

Verapamil, vascular response, differential ontogeny, rat, 278

Volume loading

effect on end-systolic pressure-volume relationship, newborn lamb, 473

left ventricular response, effect of in utero ventilation, fetal sheep, 466
Waste nitrogen, excretion, phenylacetylglutamine as vehicle for, 147

Weaning, prematurc, neonatal tolerance to hyperoxia and, rat pup, 376

Zellweger syndrome

bile acid formation. pcroxisome role, 64

22-kD peroxisomal integral membrane protein, intracellular localization, 141

Zinc, deficicncy, early dietary, laboratory assessment, weaning rat. 391 



\title{
PEDIATRIC RESEARCH
}

An International Jounal of Clinical, Laboratory, and Developmental Investigation

\author{
Volume 29 \\ January to Junc $199 \mathrm{I}$ \\ Editor-in-Chief \\ DFNNIS M. BIER \\ European Chicf Editor \\ Pieter J. J. Saler \\ American Editors
}

F. SFSSIONS COI.F

DAN M. GRANOFF

ARNOLU W. SIRAUSS

DAVID H. PERLMUIIIER

AI.AN L. SCHWART'

European Editors

(:. JAN BRUINING

STENVERT' L. S. Drop

JOHN MYSS

Herman J. Neijens

Board of Trustees

International Pediatric Research Foundation, Inc.

Gabrifi, DUC

REBFCCA H. BUCKI.FY

AI.AN H. JOBE:
LARRY J. SHAPIRO

KARI O. RAIVIO

HANS $T$. VERSMOLI)

JOSFPH B. WARSHAW

\author{
Managing Editor \\ Julia IIIldebrandt
}

Editorial Office: (314) 454-2001

Fax: (314) 367-3765

Flectronic Mail: PEIRES@WUMS

Submit manuscripts to:

Demnis M. Bier

Department of Pediatrics

Washington University

St. Louis Children's Hospital

400 South Kingshighway Blyd.

St. Louis, Missouri 63110

\section{In Europe submit to:}

Pieter J. J. Sauer

Department of Pediatrics

Erasmus University Rotterdam

Sophia Children's Hospital

Gordelweg 160

3038 GE. Rotterdam

The Netherlands

Published monthly by Williams \& Wilkins Company for the Intermational Pediatric Research Foundation Inc., and sponsored by:

American Pediatric Society

European Society for Paediatric Research

Society for Pediatric Rescarch 
